\title{
Characterization of Designed and Constructed Optical System
}

\author{
Article by Eric Amankwa \\ Ph.D Hemmo Tuovinen, Finland \\ Email:-amankwaeric@yahoo.com
}

\begin{abstract}
This laboratory work emphasize on the characterization of designed and constructed grating spectrometer. This was done by measuring the diffraction efficiency of the fabricated grating. The fabricated grating is a binary grating with height $650 \mathrm{~nm}$, period $880 \mathrm{~nm}$ and fill factor $0.40 \mathrm{~nm}$. The light source used were laser sources and halogen lamp with wavelength range $532 \mathrm{~nm}$ and $473 \mathrm{~nm}$. CCD line camera is used as a detector having 3648 pixels with each pixel having a size of $8 \mu \mathrm{m}$. The diffraction efficiencies and filter transmittance measured with the Perkin Lambda 18 spectrophotometer are compared with the constructed spectrometer. The characterization is done by measuring the full width half maximum (FWHM) of the detected laser line peaks by the constructed spectrometer.
\end{abstract}

\section{Introduction}

Spectroscopy is the study of the principles of light -matter interaction or the science of analyzing and interpreting a spectra. Generally spectroscopy is a term used to describe the interactions of various types of electromagnetic radiation with matter and it involves the analysis of light over a range of wavelengths such as wavelength of visible light. The radiation interacts with matter is directly dependent on the energy of the radiation. In spectroscopy electromagnetic wave incident on a sample characterize light after sample and characterize change in sample. The sample is characterize depending on the amount of light absorbed, emitted or the optical rotation. Absorption is the change in intensity of incident light (transmission) at different frequency or wavelength. Emission includes fluorescence, phosphorescence or raman scattering and optical rotation is the change of light incident on the sample (rotation of polarization) [1]. The main instrument use in spectroscopy is the spectrometer. A spectrometer is a device that is used to measure the properties of light emitted by a source or spectrometer is an instrument used to analyse the nature of light emitted by various sources depending on properties such as refraction, absorption, reflection and interference. The main idea in spectrometer is to split light into its components wavelength, generate a spectrum and determines its physical and chemical properties of materials. The principle behind the operation of spectrometer is that light diffracts as it traverses from one homogeneous medium to another and the diffraction angle is a function of wavelength or frequency. Spectrometers are normally designed to measure specific wavelength range of the electromagnetic spectrum. The choice of components for the design depends on the source of light to be measured. Based on these unique features of spectrometers, they are employed in many fields of study. For example in astronomy or astrophysics, spectrometers are used to measure velocities of galactic components, composition of stars and nebula chemical makeup [2]. Diffraction grating is an optical component with a periodic structure, which splits and diffracts light into several beams traveling in different directions. The different kind of gratings used in spectrometer could be reflection grating or transmission grating. Reflection grating consist of a grating superimposed on a reflective surface whereas transmission grating consist of a grating superimposed on a transparent surface [3]. Other type of gratings could be holographic grating which is produced by interference lithography, gel grating (phase or amplitude), echelle grating (cross dispersion) and each of these could be classified into either transmission or reflection type of grating. Finally grating can also be blazed, binary, concave and sinusoidal depending on the shape of its grooves. 
South American Journal of Academic Research Special Edition May 2016

There are a number of different spectrometers, each of which is designed to achieve different goals but their operational mechanisms are similar. Every spectrometer consists of three major components, diffraction layer made of diffraction grating/glass prism, collimating and focusing lenses/mirrors and detector [4]. Fig.1.1. describe the general working principle of a spectrometer. Light from a given source enters from position (1) through fixed entrance slits (2 and 3) which specify the width of the incident beam. The thin beam of light travels the length of the housing until it reaches a lens/mirror (4), which collimates the beam. The collimated beam is then intersected by diffraction grating (5) which disperses the different wavelengths (colors) of light, because the diffraction angle is a function of wavelength. This dispersed beam is collected and focused on the detector (7) by the focusing mirror/lens (6). The properties of the light is then measured based on the scales on the detector. Most detectors are calibrated to measure the intensity or polarization state of the light beam [5]. In this laboratory work the task is to first, the efficiency of the constructed binary diffraction grating spectrometer is measured and compared with the theoretically designed values to that of the commercial spectrophotometer. The constructed spectrometer is then characterized by calculating the spectral resolution. Chapter two reviews the experimental set up, procedure and results. Chapter three reviews discussion of results and Chapter four finally reviews the conclusion part of the work.

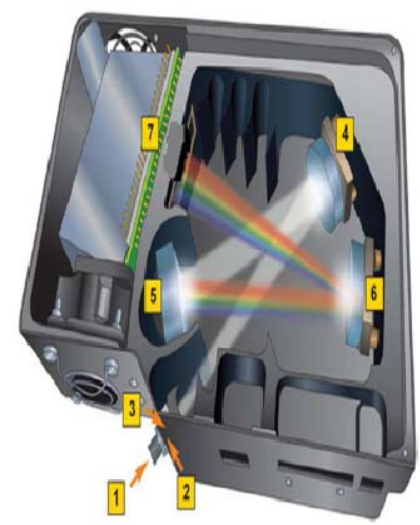

Figure 1.1: Schematic diagram of how light propagates through a commercial spectrometer [5].

\section{Experimental setup and Characterization}

This chapter reviews the equipment, experimental setup and the characterization are described in detail.

\section{Equipments}

The equipments used in the experiment includes the following:

1. Two lasers (473 $\mathrm{nm}$ and $532 \mathrm{~nm})$

2. Mirrors

3. Lenses

4. Optical breadboard

5. Power meter

6. Diffraction grating

7. Halogen lamp

8. CCD line camera

9. Holders and mounts

10. Adhesive tape

11. Personal computer

12. Spectrophotometer (PerkinElmer Lambda 18) 
13. Tool kits (balldrivers, screwdrivers and tap kits)

14. Attenuators

15. Beam splitter

16. Filter

\section{Diffraction efficiency measurements}

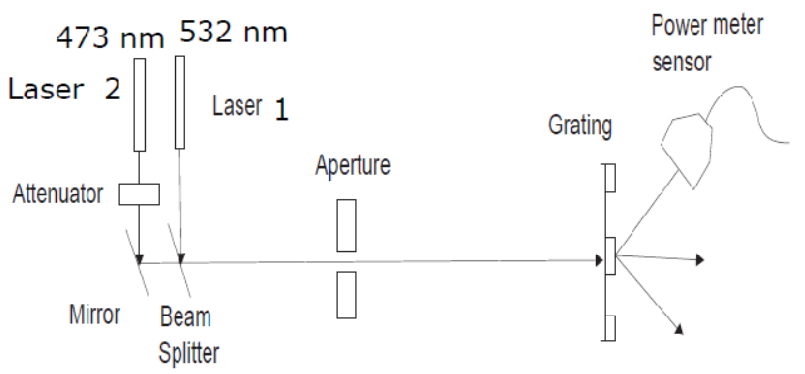

Figure 2.1: Typical diffraction efficiency measurement setup.

Fig. 2.1. depicts the diffraction efficiency measurement setup for two lasers $532 \mathrm{~nm}$ and $473 \mathrm{~nm} .532 \mathrm{~nm}$ laser which is TM polarised is turned on and the beam is directed to the power sensor of the power meter through attenuator, mirrors, aperture and diffraction grating as shown in Fig. 2.1. It reviews that, there was a reduction in power as the beam passes through the attenuator. First the power is measured without using diffraction grating, later is placed in the holder and the power is measured for the diffraction orders, $-1,0$ and +1 . The grating diffraction efficiency which is the ratio of the power of diffracted light beam at a given order to that of the incident power of the beam. It is dimensionless quantity and is given by the relation:

$$
\eta_{m}(\lambda)=\frac{I_{m}(\lambda)}{I_{o}(\lambda)} \times 100 \% .
$$

$I_{\mathrm{m}}(\lambda)$ is the intensity of the mth order and $I_{o}(\lambda)$ is the incident beam intensity without diffraction grating. The experiment is repeated for the $473 \mathrm{~nm}$ laser which is TE polarised and the diffraction efficiency is calculated using Eq. 2.1. and the results were tabulated in chapter three. One should note that, the diffraction efficiency depends on the following factors and parameters: polarization state, spectral range of light, material from which the grating is made, grating period, fill factor and groove height [3]. PerkinElmer Lambda 18 Commercial spectrophotometer was used to measure the diffraction efficiency of the grating within the wavelength limit of $440 \mathrm{~nm}-540 \mathrm{~nm}$ and the results obtained are compared in chapter three.

\section{Spectral resolution measurement}

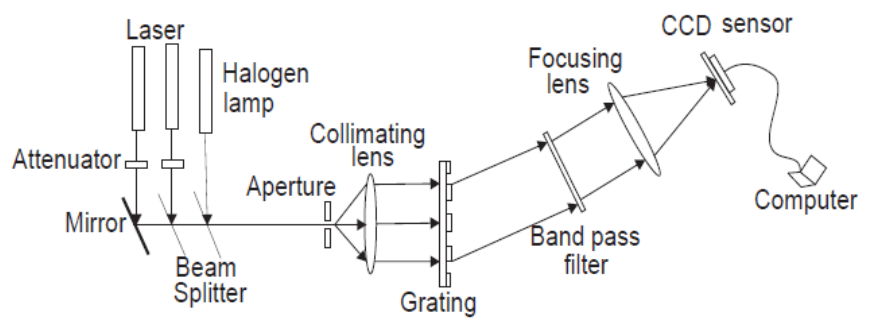

Figure 2.2: Spectrometer characterization setup.

Fig. 2.2. shows spectral resolution or characterization measurement setup which is given by the relation: 
South American Journal of Academic Research

Special Edition May 2016

$$
\delta(\lambda)=\frac{R F \times \Delta \lambda \times W_{s}}{n \times W_{p}},
$$

where $\delta \lambda$ is the minimum difference of wavelength that the system can resolve, RF is the resolution factor that is the number of pixels acquired by one spectral line at full width half maximum (FWHM) of the laser peak, $\Delta \lambda$ is the range of wavelength, $440 \mathrm{~nm}-540 \mathrm{~nm} . n$ is the number of pixels contained in the whole spectrum. $W_{\mathrm{s}}$ and $W_{\mathrm{p}}$ are the slit and pixel width equivalent to $8 \mu m$ in this task. The calculation of both $R F$ and $n$ one can obtain the spectral resolution of the constructed spectrometer. The procedure of the experimental setup is that, the attenuation of the laser beam power, distance of the lens to that of the detector, tilt of the mirrors and the diffraction grating were carefully optimized to achieve narrow laser line peak because the sharper the peak the most relevant or best the spectral resolution and the best constructed spectrometer. For scattering (dispersive) array spectrometers one can determine the spectral resolution by considering three components, the grating sets the limit of the wavelength, detector whose pixel width sets the lower limit of spatial resolution and the slit which determine the minimum image size formed on the detector plane [6,7]. The number of pixels per nm were estimated from the two lasers, the difference of the number of pixels $n$ at $440 \mathrm{~nm}$ and $540 \mathrm{~nm}$ were also estimated and the difference of the number of pixels at the midpoints of the laser peaks were also estimated which yields the full width half maximum (FWHM) or the resolution factor (RF) whose power is the ability of the spectrometer to segregate the adjacent lines in the spectrum [3]. Ultimately filter was inserted between the grating and the lens to measure the transmittance and the following parameters were used for the binary diffraction grating, achromatic doublet lens of $100 \mathrm{~mm}$ focal length, grating height of $650 \mathrm{~nm}$, period of $880 \mathrm{~nm}$ and fill factor of 0.4. The light sources used were blue and green laser respectively as $473 \mathrm{~nm}$ and $532 \mathrm{~nm}$.

\section{Results}

This chapter reviews the results obtained in the laboratory experiment compared to the theoretical calculations and design.

\section{Diffraction efficiency measurement result}

Table 3.1 Diffraction efficiency measurement with lasers.

\begin{tabular}{|l|l|l|l|l|}
\hline & \multicolumn{2}{|l|}{$473 \mathrm{~nm}$ Laser } & \multicolumn{2}{l|}{$532 \mathrm{~nm}$ Laser } \\
\hline $\mathrm{m}$ & $P_{m}(\lambda) \mathrm{mW}$ & $\eta_{m}(\lambda)$ & $P_{m}(\lambda) \mathrm{mW}$ & $\eta_{m}(\lambda)$ \\
\hline-1 & 0.62 & $30.2 \%$ & 0.96 & $40.1 \%$ \\
\hline 0 & 0.31 & $15.1 \%$ & 0.19 & $7.9 \%$ \\
\hline+1 & 0.63 & $30.7 \%$ & 0.97 & $40.6 \%$ \\
\hline
\end{tabular}

The incident light intensities were measured for lasers $473 \mathrm{~nm}$ and $532 \mathrm{~nm}$ with-out diffraction grating. Respective values obtained are $2.05 \mu \mathrm{W}$ and $2.39 \mu \mathrm{W}$. The power is measured at different diffraction orders for $-1,0$ and +1 and efficiencies calculated as shown in table 3.1. The blue curve in Fig. 3.3. represents the measurement results of the PerkinElmer Lambda 18 Spectrophotometer and the asterisks represent the measurement results with the lasers. Fig. 3. 2. represent the design diffraction efficiency of the grating (theoretical design). The average efficiency was found to be about $30.1 \%$ by the measurement with the spectrophotometer and the peak efficiency at $540 \mathrm{~nm}$ was obtained to be around $36.0 \% .473$ $\mathrm{nm}$ the efficiency was found to be $26.2 \%$ and at $532 \mathrm{~nm}$ the efficiency was found to be about $35.2 \%$. Efficiencies measured using the lasers, at $473 \mathrm{~nm}$ the efficiency was found to be about $30.2 \%$ and at $532 \mathrm{~nm}$ the efficiency was found to be around $40.1 \%$. Difference of about $4.0 \%$ at $473 \mathrm{~nm}$ were observed for both measurement. Difference of about $4.9 \%$ at $532 \mathrm{~nm}$ were observed for both measurement. This is due to the polarization of the laser light because the light source of the spectrophotometer is unpolarised, difficulty in positioning the grating perfectly, the use of the card to block the unwanted orders of diffraction some portion of the 
diffracted light was not collected by the integrating sphere inside the spectrophotometer and finally the PerkinElmer Lambda 18 Spectrophotometer is constructed carefully by professionals and very costly device which took a lot of time to built completely. The transmittance between $440 \mathrm{~nm}$ and $460 \mathrm{~nm}$ was very low because there was a drop in the diffraction efficiency at $440 \mathrm{~nm}$.

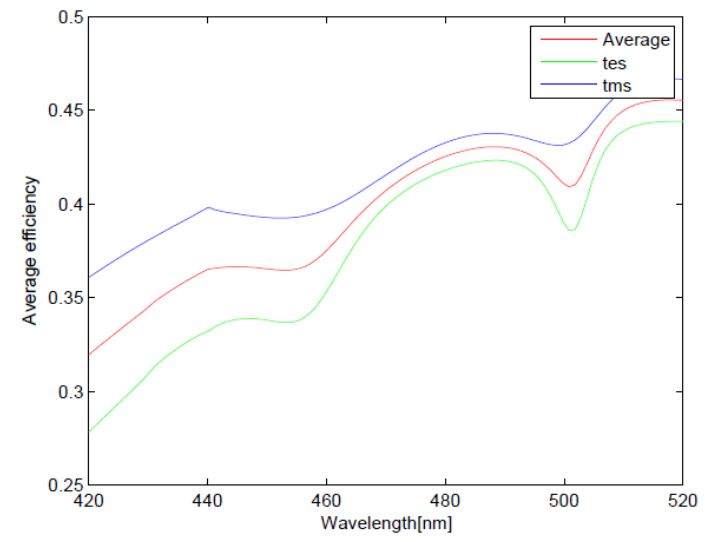

Figure 3.1: Average Efficiency of TE and TM polarization.

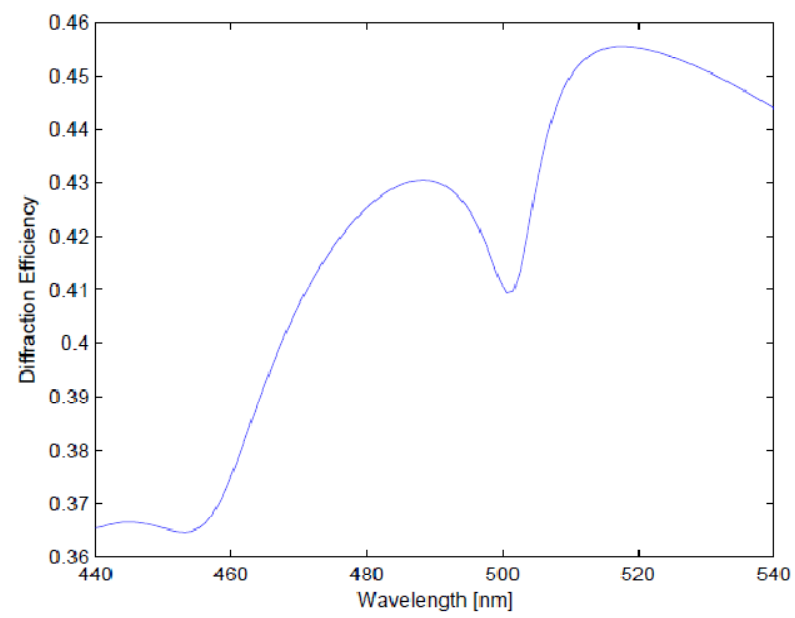

Figure 3.2: Average Efficiency of TE and TM polarization.

\section{Spectral resolution measurement results}

The task is to calculate the number of pixels in the whole spectrum (n). One can consider first the pixels per nm after then pixels at $440 \mathrm{~nm}$ and $540 \mathrm{~nm}$ respectively. The pixels of 473 $\mathrm{nm}$ resulted at 1411 pixels and the pixels of $532 \mathrm{~nm}$ resulted at 2722 pixels. The full width half maximum which is the resolution factor is obtained approximately as 3.5 pixels graphically by a matlab ginput command or numerically by curve fitting as shown in fig. 3.5.

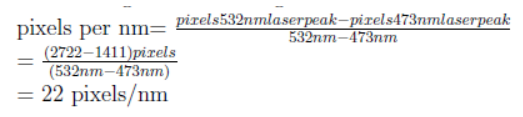


South American Journal of Academic Research

Special Edition May 2016

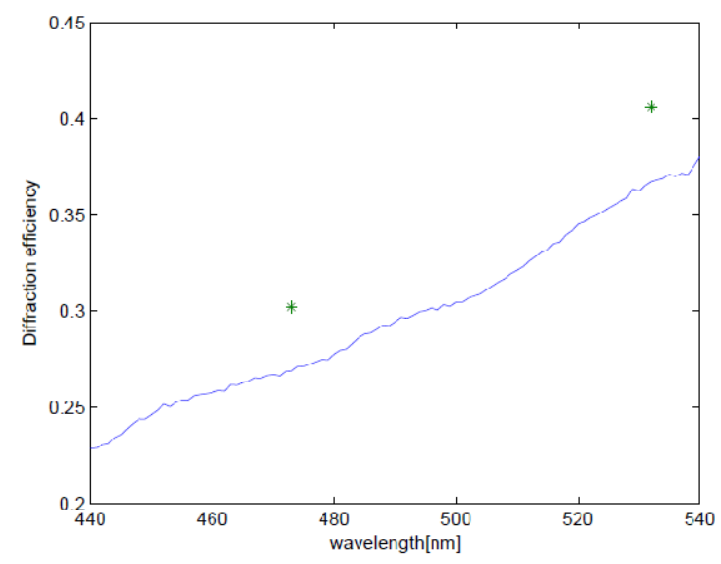

Figure 3.3: Diffraction efficiency measurement results.

Therefore ; pixels at $440 \mathrm{~nm}=$ pixels at $473 \mathrm{~nm}-40 \mathrm{~nm} \times 22$ pixels $/ \mathrm{nm}$ $=(1411-40 \times 22)$ pixels

$=531$ pixels

Again; pixels at $540 \mathrm{~nm}=$ pixels at $532 \mathrm{~nm}+8 \mathrm{~nm} \times 22$ pixels $/ \mathrm{nm}$

$=(2722+8 \times 22)$ pixels

$=2898$ pixels

Finally; $\mathrm{n}$ can be calculated as , $\mathrm{n}=$ pixels at $540 \mathrm{~nm}$ - pixels at $440 \mathrm{~nm}$

$=(2898-531)$ pixels

$=2367$ pixels

substituting the values into equation 2.2, the spectral resolution

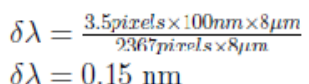

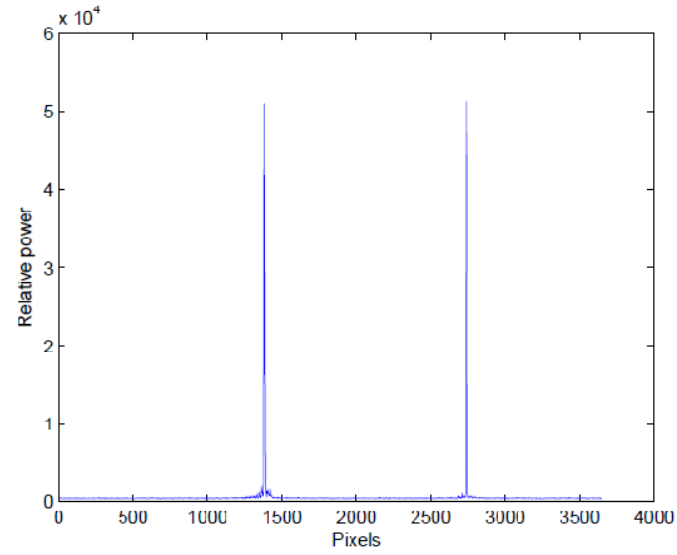

Figure 3.4: Laser line peaks measured by CCD line camera .

Therefore the constructed and designed spectrometer has a resolving power of about 0.15 $\mathrm{nm}$ which is 2.67 times compared to the theoretical calculations and design. Difference of about $0.25 \mathrm{~nm}$. This difference could be due to irregularities in the grating period due to limitations on the fabrication, inaccurate positioning of the lens and detector, polarization of the light sources (lasers) and lastly other reason could be aberration of the lens which was not taken into account during the design phase but the aberration appears during practical arrangements. Finally resolving power of $0.8 \mathrm{~nm}$ is obtained at a wavelength of $540 \mathrm{~nm}$. 


\section{Green filter transmittance measurement}

Transmittance of a green filter was measured using Perkin Lambda 18 spectrometer and with the constructed spectrometer so that the results could be compared. First the results of the constructed spectrometer were converted to wavelength $(\mathrm{nm})$ since the results were in pixel scale within the range of 1 to 3648 pixels. Fig. 3.6. illustrates the halogen lamp spectrum measured by the constructed spectrometer whereas Fig. 3.7. illustrates halogen lamp with a filter. The normalised transmittance was done by dividing the spectrum of the green filter by the halogen lamp. Fig. 3.8. illustrates comparison of normalised transmittance results of constructed spectrometer and spectrophotometer. The spectrometer has a transmittance of about $48 \%$ compared to the spectrophotometer with a transmittance of about $52 \%$ almost a difference of $4 \%$ for both. This shows that the constructed spectrometer gave a reasonable measurement of the transmittance. There was a low level of noise between $440 \mathrm{~nm}$ and 540 $\mathrm{nm}$ because signal to noise increased as the signal level increased while the noise remained constant. The noise at $440 \mathrm{~nm}$ is higher compared to $540 \mathrm{~nm}$ because the signal is less at the former point than the latter and the signal to noise ratio is inversely proportional to the signal level.

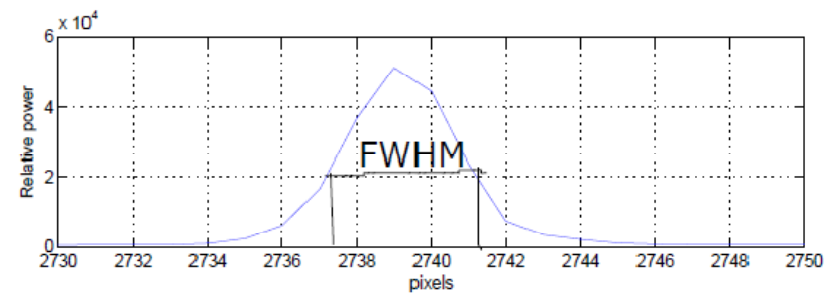

Figure 3.5: Full width half maximum (FWHM) at $532 \mathrm{~nm}$ laser line.

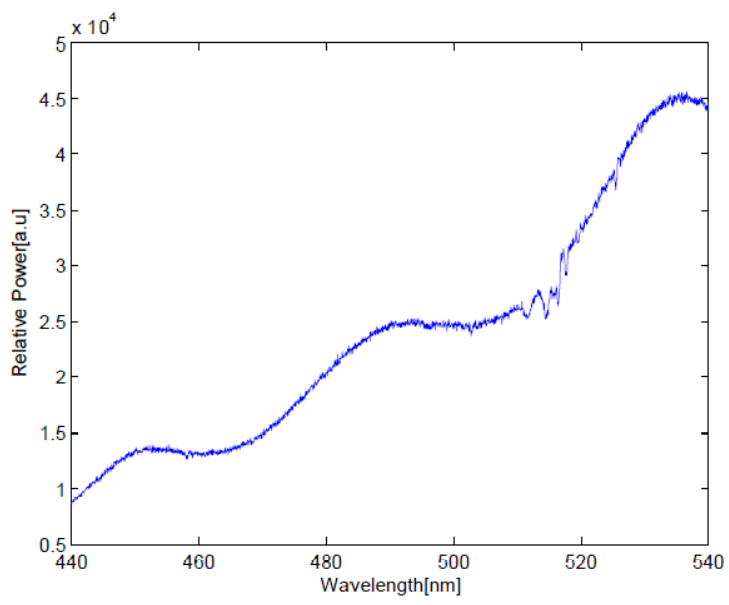

Figure 3.6: Halogen lamp spectrum. 
South American Journal of Academic Research Special Edition May 2016

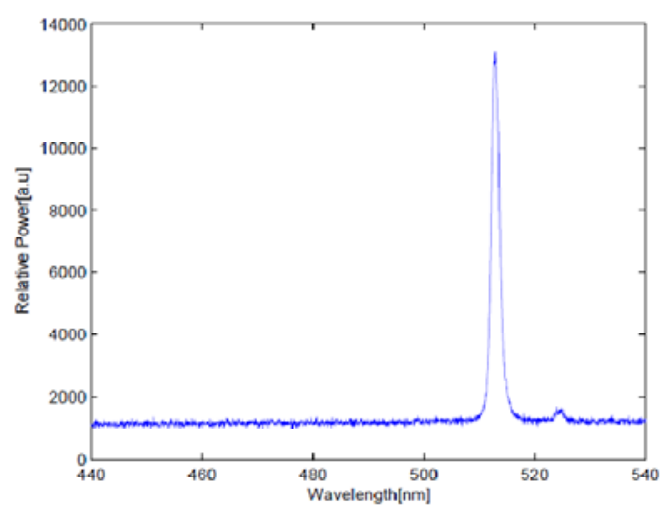

Figure 3.7: Halogen lamp spectrum with a filter.

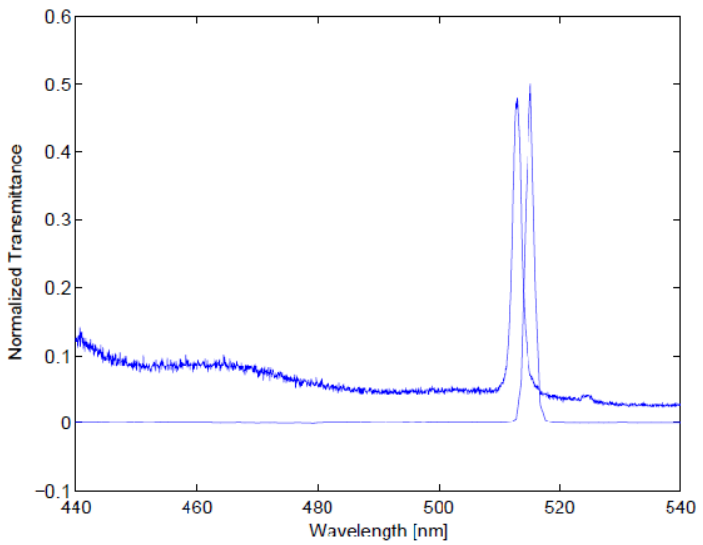

Figure 3.8: Comparison of normalized transmittance results of constructed spectrometer and spectrophotometer.

\section{Conclusions}

A constructed transmission grating spectrometer has been characterized in this laboratory work. The characterization was done by measuring the diffraction efficiency using lasers as light source and power meter as a measuring device. The results were compared with the spectrophotometer. The following results were obtained using the spectrophotometer, efficiency of $36 \%$ at $532 \mathrm{~nm}$ and $26.2 \%$ efficiency at $473 \mathrm{~nm}$ for the first minus diffraction order. The constructed spectrometer, the values obtained for the first minus order diffraction efficiencies were $30.2 \%$ at $473 \mathrm{~nm}$ and $40.1 \%$ at $532 \mathrm{~nm}$ with difference of $4.0 \%$ and $4.1 \%$ for both. Spectral resolution of $0.15 \mathrm{~nm}$ resulted which is 2.67 times the design, a difference of about $0.25 \mathrm{~nm}$ and filter transmittance of the spectrometer resulted $48 \%$ and that of the spectrophotometer resulted $52 \%$ almost a difference of $4 \%$ for both. Finally the results obtained shows that the spectrometer gave reasonable results and therefore performed well.

\section{Bibliography}

[1] J. M. Hollas, Modern Spectroscopy, 4 ed. (John Wiley and sons Limited, West Sussex, 2004).

[2] R. S. Winsor, J. W. Mackenty, M. Stiavelli, M. A. Greenhouse, J. E. Mentzell, R. G. ohi IV, and R.

F. Green, "Optical design for an infrared imaging multi-object spectrometer(IRMOS)," PROC-SPIE 4092, 102-108 (2000).

[3] C. Palmer, Diffraction Grating Handbook, 2nd ed. (Wiley, New York, 1997).

[4] U. Platt and J. Stutz, Differential Optical Absorption Spectroscopy (Springer Berlin Heidelberg, 2008).

[5] http:www.oceanoptics.com/products/benchoptics ge.asp, 
South American Journal of Academic Research Special Edition May 2016

[6] H. Tuovinen, "Characterization of designed and constructed optical system," University of Eastern Finland, Joensuu (2015).

[7] bwtek, http://bwtek.com/spectrometer-part-5-spectral-resolution/ (valid 2015). 